Bucaramanga, Santander, Colombia; ${ }^{3}$ Corporación Autonoma Regional para la Defensa de la Meseta de Bucaramanga, Bucaramanga, Santander, Colombia

A previous study in Bucaramanga, Colombia had shown that there were not association between outdoor air pollution and incidence of respiratory symptoms related to asthma in healthy paediatric population. We investigated whether exposure to different levels of outdoor air pollution are associated to incidence of respiratory symptoms in population with chronic diseases. Three pollution zones were selected according to historic measures of particulate matter $<10 \mu \mathrm{g} / \mathrm{m}^{3}$ (PM10): low $\left(<40 \mu \mathrm{g} / \mathrm{m}^{3}\right)$, medium $(40-60 \mu \mathrm{g} /$ $\left.\mathrm{m}^{3}\right)$ and high $\left(>60 \mu \mathrm{g} / \mathrm{m}^{3}\right)$. A total of 756 patients with chronic cardiovascular and respiratory disease were selected around the air quality stations at each zone. This was a cohort study with the follow-up-phase lasting 6 months using daily calendar of symptoms and clinic visits. Symptoms with higher incidence rates were sneeze and hacking cough. Incidence rate of total symptoms per 100 -participant-day was 46 with differences between zones. Incidence rate ratio of total symptoms between low and high zone was 1.14 (95\% CI 1.11 to 1.16). Except for wheezing (IRR 0.84; 95\% CI 0.78 to 0.91 ) and inhalers use (IRR $0.68 ; 95 \%$ CI 0.64 to 0.73 ), all symptoms were higher in middle pollution area, but multivariate analysis using Poisson multilevel approach shown after adjustment for confounding variables, high pollution area is associate with $64 \%$ and $77 \%$ more symptoms compared with middle and low pollution area, respectively. These results suggest that in populations with morbidity outdoor air pollution is a key determinant of respiratory symptoms and respiratory negative effects are seen over $60 \mu \mathrm{g} / \mathrm{m}^{3}$.

\section{P2-467 TWO-SIDED NUTRITIONAL PROBLEMS AMONG SCHOOL- AGED CHILDREN IN VIETNAM}

doi:10.1136/jech.2011.142976l.95

${ }^{1} \mathrm{~N}$ Sakamoto, ${ }^{1} \mathrm{~L}$ Yang, ${ }^{2} \mathrm{P}$ T T Hoa, ${ }^{2} \mathrm{~L}$ T Hop. ${ }^{1}$ National Research Institute for Child Health \& Development, Setagaya, Tokyo, Japan; ${ }^{2}$ National Institute of Nutrition, Hanoi, Viet Nam

Objective To estimate the prevalence of underweight, overweight and obesity in Vietnamese children and adolescents aged 6-18 years in both urban and rural areas.

Methods A cross-sectional study was conducted in 2006. Data on height and weight of 6354 children living in rural areas and 5280 children in urban areas were used for analysis. The prevalence of underweight/thinness, overweight and obesity was estimated according to the United States Centers for Disease Control (CDC) growth charts and WHO child growth standards (WHO Reference 2007).

Results In urban areas, the prevalence of underweight, overweight and obesity among children and adolescents aged 6-18 years was $9.5 \%, 21.0 \%$ and $8.4 \%$ in boys and $10.0 \%, 9.7 \%$ and 1.8 in girls, respectively, based on the CDC cut-offs. In rural areas, the corresponding rates were $26.2 \%, 1.2 \%$ and $0.3 \%$ in boys and $20.4 \%, 0.7 \%$ and $0.1 \%$ in girls, respectively. Urban children were more likely to be overweight than rural children. Conversely, rural children were more likely to be underweight than urban children.

Conclusions The co-occurrence of overweight and underweight among urban children and adolescents and persistent underweight epidemic among rural peers are the main health concerns in Vietnam. Policy planner should develop appropriate health strategies for urban populations to reduce the rising epidemic of over nutrition, while also focusing on the needs underweight children. In rural areas, it the government should provide more effective intervention to reduce poverty and improve the nutrition status of rural children.

\section{P2-468 INEOUITIES IN THE EARLY CONDITIONS OF LIFE: EFFECTS ON THE MORTALITY OF ELDERS. RESULTS FROM THE SABE STUDY - SÃO PAULO - BRASIL: 2000-2006}

doi:10.1136/jech.2011.142976l.96

1J L F Santos, ${ }^{2} \mathrm{M}$ L Lebrão, ${ }^{3} Y$ A 0 Duarte. ${ }^{1}$ Faculdade de Medicina de Ribeirão Preto-USP, Ribeirão Preto, São Paulo, Brazil; ${ }^{2}$ Faculdade de Saúde Pública-USP, São Paulo, São Paulo, Brazil; ${ }^{3}$ Escola de Enfermagem-USP, São Paulo, São Paulo, Brazil

Introduction Studies indicate connections between childhood conditions and health in old ages. Early conditions of life may be related to individual factors of development. Thus, rural areas can harbour states of child development different from those in the urban ambience.

Objective To evaluate the impact of rural origin on mortality of elders living in a urban region.

Methods Data are from SABE: a longitudinal survey in São Paulo-2000/2006. The explanatory variable "origin", was obtained from the question: "Have you lived in the countryside for more than 5 years before the age 15 ?" Control variables are sex, age, education, income; having had malaria, pneumonia or typhoid fever before the age of 15. Outcome was "death": people alive in $2000(n=2143)$ and confirmed dead before or during the second round in $2006(n=649)$.

Results Rao-Scott tests showed differences according to the origin of the elders for all variables except age and typhoid fever. A Poisson regression was applied to evaluate the associations between "origin" and "death" in the presence of all other variables, controlling for time of exposure. Incidence Rate Ratio (IRR) for rural origin was $1.54(p=0.03)$. That is, having lived in the countryside before the age of 15 increased the death rate by $54 \%$. Sex, age and income were also significant, with IRR of $1.79 ; 3.57$ and 1.69 .

Conclusions These results demonstrate that inequities such as ruralurban inequality, even occurring in the young ages, can perpetuate differences through a person's life, including an increased chance of dying when older.

\section{P2-469 MATERNAL VITAMIN D STATUS AND DELIVERY BY CESAREAN}

doi:10.1136/jech.2011.142976l.97

T Scholl, ${ }^{*} X$ Chen, M Schleuter, T P Stein. University Medicine and Dentistry New Jersey, Stratford, New Jersey, USA

Introduction Factors which increase risk of delivery by cesarean include older maternal age, obesity, nulliparity, minority status and a prior history of cesarean delivery. In all probability less well defined factors exist at least one of which may be nutritional-maternal vitamin $\mathrm{D}$ deficiency and insufficiency during pregnancy. We examined the association of circulating levels of vitamin $\mathrm{D}$ to risk of cesarean delivery using prospective data.

Method Circulating maternal 25-hydroxyvitamin D and intact parathyroid hormone at entry to care (13.8 \pm 0.17 weeks - mean, SEM) were assayed by HPLC (25 hydroxyvitamin D) and radioimmunoassay (parathyroid hormone) in a cohort of 1153 low income and minority gravidae from Camden, NJ.

Results The prevalence of vitamin D deficiency (19.8\%) and insufficiency $(50.5 \%)$ at entry was high and accompanied by increasing concentrations of parathyroid hormone, a functional indicator of vitamin D status. Risk for primary cesarean, for secondary cesarean, as well as for all deliveries by cesarean was increased approximately twofold for vitamin $\mathrm{D}$ deficient women (<37.5 nmol/1) except for primary cesarean delivery where risk also was increased 1.5-fold with levels suggestive of insufficiency $(37.5-80 \mathrm{nmol} / \mathrm{l})$. Vitamin D deficiency was linked to indications for cesarean including a twofold 